\title{
Comparison of Deferoxamine, Deferiprone and Deferasirox Iron-Chelating Agents in Reducing Serum Ferritin Levels in Patients with Thalassemia Major
}

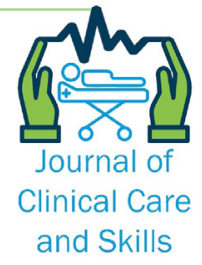

\section{ARTICLE INFO}

\section{Article Type}

Descriptive Study

\section{Authors}

Arya A. ${ }^{1} M D$,

Jokar S. ${ }^{1} M D$,

Etemadfar $\mathrm{P}^{2} M D$,

Malekzadeh J.M. ${ }^{3} P h D$,

Jannesar R. ${ }^{4} M D$,

Rohani M. ${ }^{5} M D$,

Mohamadi T. ${ }^{4}$ SS

Kharaman F. ${ }^{6} M S c$,

Yosefi M. ${ }^{6} M S c$,

Hatamipour S. ${ }^{5} M D$,

Roozbehi A. ${ }^{* 7} M D$

How to cite this article
Arya A, Jokar S, Etemadfar P, Malekzadeh
J.M, Jannesar R, Rohani M, Mohamadi T,
Kharaman F, Yosefi M, Hatamipour S, Roo-
zbehi A. Comparison of Deferoxamine,
Deferiprone and Deferasirox Iron-Chelati-
ng Agentsin Reducing Serum Ferritin Lev-
els in Patients with Thalassemia Major
Journal of Clinical Care and Skills. 2020;1(
4):189-193.

${ }^{1}$ Internal Medicine Department, Medicine Faculty, Yasuj University of Medical Sciences, Yasuj, Iran

${ }^{2}$ Paediatric Hematology \& Oncology Department, Medicine Faculty, Yasuj University of Medical Sciences, Yasuj, Iran

${ }^{3}$ Nutrition Department, Health Sciences Faculty, Yasuj University of Medical Sciences, Yasuj, Iran

${ }^{4}$ Molecular Microbiology Department, Dena Pathobiology Laboratory, Yasuj, Iran ${ }^{5}$ Obstetrics \& Gynecology Department, Medicine Faculty, Yasuj University of Medical Sciences, Yasuj, Iran

${ }^{6}$ Nursing Care Faculty, Yasuj University of Medical Sciences, Yasuj, Iran

${ }^{7}$ Anatomy Department, Medicine Faculty, Yasuj University of Medical Sciences, Yasuj, Iran

\section{*Correspondence}

Address: Medicine Faculty, Yasuj University of Medical Sciences, Yasuj, Iran.

Phone: +98 (74) 223330700

Fax: -

amroozbehi@gmail.com

\section{Article History}

Received: December 11, 2019

Accepted: February 29, 2020

ePublished: October 01, 2020

\section{A B S T R A C T}

Aims Patients with thalassemia major suffer from iron overload due to excessive cell lysis. Iron-chelating agents are used to preventing the effects of iron overload on the body. Deferoxamine, deferiprone, and deferasirox are the most common iron-chelating agents. The aim of this study was to compare deferoxamine, deferiprone, and deferasirox iron-chelating agents in reducing serum ferritin levels in patients with thalassemia major.

Instrument \& Methods This descriptive cross-sectional study was performed on 114 thalassemia major patients in the Cooley's ward of Shahid Beheshti Hospital, Yasuj, Iran, in 2018 who had been taking iron supplements for the past year. Census sampling was done. After recording patients' information, such as age, sex, and type of iron used, the blood sample was taken from the patients to measure serum ferritin and the results were analyzed using SPSS 22 software and multivariate analysis of variance.

Findings The mean serum ferritin level was $3438.70 \pm 2872.88 \mathrm{ng} / \mathrm{ml} .14$ patients were taking deferoxamine, 62 patients were using deferiprone, and 38 patients were consuming deferasirox. There was no significant difference between iron-chelating agents in reducing ferritin levels ( $p>0.05)$. Also, there was no significant difference between males and females in terms of decreased ferritin levels ( $p>0.05$ ).

Conclusion There is no difference between deferoxamine, deferiprone and deferasirox ironchelating agents in reducing the ferritin levels of patients with thalassemia and all can be administrated considering the cost, availability, condition, and patient's desire.

Keywords Thalassemia Major; Iron Chelating Agents; Deferoxamine; Deferiprone; Deferasirox; Ferritin

\section{CIT A T I O N L INKS}

[1] Beta-thalassemia ... [2] The clinical sequelae of intravascular hemolysis and extracellular plasma hemoglobin: a novel ... [3] Defining autoimmune hemolytic anemia: a systematic review of the terminology ... [4] Durch $\beta$-Thalassaemia minor maskierte autoimmune perniziöse ... [5] How I treat transfusional iron ... [6] Harrison's principles of internal ... [7] Pathophysiology of ... [8] A phase 3 study of deferasirox (ICL670), a once-daily oral iron chelator, in patients ... [9] Pharmacokinetics and renal elimination of desferrioxamine and ferrioxamine in healthy subjects ... [10] Safety, tolerability, and pharmacokinetics of ICL670, a new orally active ironchelating agent in patients with transfusion-dependent iron overload ... [11] Current approach to iron chelation ... [12] Cross-talk between available guidelines for the management of patients with ... [13] Light and shadows in the iron chelation treatment ... [14] Improved treatment satisfaction and convenience with deferasirox in iron-overloaded patients with ... [15] Accuracy of magnetic resonance imaging in diagnosis of liver iron overload ... [16] Iron chelation in thalassemia ... [17] Oral chelators deferasirox and deferiprone for transfusional iron overload in thalassemia ... [18] Efficacy of deferasirox in reducing and preventing cardiac iron overload in ... [19] Iron overload in thalassemia and related conditions: therapeutic goals and assessment of ... [20] Long-term efficacy of oral deferiprone in management of iron overload in ... [21] Cardiac morbidity and mortality in deferoxamine-or deferiprone-treated patients ... [22] Deferiprone versus deferoxamine in patients with thalassemia major ... [23] Comparison between desferrioxamine and combined therapy with desferrioxamine and deferiprone... [24] Comparing the efficacy of Dexeroyx (Osveral) and Deferoxamine ... [25] Survival and complications in patients with thalassemia major treated ... 


\section{Introduction}

Thalassemia is a genetic disorder characterized by excessive lysis of red blood cells due to a defect in the hemoglobin beta chain and hemoglobin instability ${ }^{[1] .}$ Most of the body's iron is in the hemoglobin, and in cases of the red blood cell lysis, the iron inside the patients develop anemia or iron overload dependent on the type of lysis [2]. In general, intravascular hemolysis and extravascular hemolysis are two forms of red blood cell lysis [3]. In intravascular hemolysis, hemoglobin is lysed in the arteries, and as a result, the iron is excreted in the urine, and patients develop anemia, whereas, in extravascular hemolysis, iron is stored in lymphatic organs, such as the spleen and patients develop iron overload [3]. The red blood cells in thalassemia patients are subjected to extravascular hemolysis, and because the red develop iron overload [4]. Increased iron ion load in patients with thalassemia, especially thalassemia major patients who are constantly receiving blood units leads to the deposition of iron ions in various organs, such as the heart, liver, kidneys, thyroid, and parathyroid glands resulting in a defect in the function of these organs $[5,6]$. Iron deposition in the heart causes heart failure, such as cardiomyopathy or in the liver causes liver cirrhosis, which can be prevented by reducing the iron ions load in thalassemia patients [7]. Iron-chelating agents are used to preventing these side effects [5]. It is essential to evaluate and control the serum ferritin level in health decisions to reduce the side effects of iron overload, and subsequently the costs on the health system. No study has yet been conducted suggesting the indication for prescribing these iron-chelating agents in different regarding age and gender, and also no study simultaneously had compared these three drugs on serum ferritin levels in patients with thalassemia. On the other hand, providing a regional database of thalassemia major patients can be helpful in order to take iron-chelating agents regularly and a tendency toward a certain type of iron-chelating agent to start taking the drug. This study was done to investigate serum ferritin levels and compare the effects of iron-chelating agents on serum ferritin levels in patients with thalassemia major in Yasuj, Iran, who have been taking one of the deferoxamine, deferiprone and deferasirox iron-chelating agents for the past year. Therefore, the aim of this study was to compare deferoxamine, deferiprone, and deferasirox iron-chelating agents in reducing serum ferritin levels in patients with thalassemia major.

\section{Instrument and Methods}

This descriptive cross-sectional study was performed on 114 thalassemia major patients in the Cooley's ward of Shahid Beheshti Hospital, Yasuj, Iran, in 2018 who had been taking iron supplements for the past year. Census sampling was done and all patients with hemoglobin is separated from the globin chain, and blood cells are constantly lysing, these patients

thalassemia (120 patients) who have been referring to the Cooley's ward of Yasuj Hospitals to receive one or two red blood cell units monthly were included. Patients have been taking one of three iron-chelating agents of deferoxamine, deferiprone, and deferasirox, and $1 \mathrm{mg}$ of folic acid daily.

Inclusion criteria included regular intake of one of the three iron-chelating agents of deferoxamine, deferiprone, and deferasirox, no history of cardiovascular disease, liver disease, inflammatory diseases, and chronic kidney disease.

Finally, 114 eligible patients were included in the study. Six patients were excluded as they did not take iron-chelating agents properly, or their ferritin levels showed 2 standard deviations higher than the normal population, indicating that patients did not take regular chelating agents properly.

After obtaining conscious consent and determining the age, sex, and type of used iron-chelating agents (deferasirox, deferiprone, and deferoxamine), a blood sample was taken from the patients. The samples were then sent to a laboratory to measure serum ferritin levels. To measure ferritin, the Advia 30 hematology analyzer (Siemens; Germany) and the FERRITIN 250T CENTAUR kit (Siemens; Germany) were used.

SPSS 22 software and multivariate analysis of variance were used to analyze the data.

\section{Findings}

Of a total of 114 patients, 53 patients (46.5\%) were male and 61 patients (53.5\%) were female. The age range of the patients was 3-38 years with the mean age of was $20.56 \pm 8.03$ years old.

The average ferritin level was $3438.70 \pm 2872.88$ $\mathrm{ng} / \mathrm{ml}$ and $51.7 \%$ of the patients had a ferritin level of $<2500 \mathrm{ng} / \mathrm{ml}$ and $48.2 \%$ had a ferritin level of $>2500$ $\mathrm{ng} / \mathrm{ml}$ (Table 1).

Among the patients, 14 cases reported using deferoxamine, 62 cases deferiprone, and 38 cases deferasirox.

There was no significant difference between ironchelating agents in reducing ferritin levels ( $p>0.05)$. Also, there was no significant difference between male and female patients in terms of ferritin levels ( $>0.05$; Table 2).

Table 1) The absolute and relative frequency of patients according to serum ferritin level $(\mathrm{n}=114)$

\begin{tabular}{llc}
\hline \multicolumn{1}{c}{ Ferritin level $(\mathrm{ng} / \mathrm{ml})$} & No. & \% \\
\hline Lower than 1000 & 26 & 22.8 \\
$1000-2500$ & 33 & 28.9 \\
Over 2500 & 55 & 48.2 \\
\hline
\end{tabular}

Table 2) Comparison of the mean ferritin levels $(\mathrm{ng} / \mathrm{ml})$ according to sex and the type of iron-chelating agents

\begin{tabular}{lcc}
\multicolumn{1}{c}{ Variables } & Mean ferritin levels & P. value \\
\hline Gender & $3282.23 \pm 2725.54$ & \\
Male & $3574.65 \pm 3010.90$ & 0.51 \\
Female & & \\
Iron-chelating agents & \\
Deferoxamine & $2867.63 \pm 2623.57$ & \\
Deferiprone & $3647.35 \pm 3071.09$ & 0.44 \\
Deferasirox & $3308.68 \pm 2650.49$ & \\
\hline
\end{tabular}




\section{Discussion}

This research was the first study to compare serum ferritin levels in patients with thalassemia major who have routinely been taking one of the three ironchelating agents of deferoxamine, deferiprone, or deferasirox. Of the 114 eligible patients, 14 cases reported using deferoxamine, 62 cases deferiprone, and 38 cases deferasirox.

In this study, the majority of patients reported using deferiprone, whereas deferoxamine was less common. After deferiprone, deferasirox was the most used drug. Considering the cost, difficulty of injection, and availability, our study was similar to other studies, as deferiprone was more popular due to its oral administration, availability, and costeffectiveness. To administrate an iron-chelating agent in a patient at the risk of increased iron load, various factors, such as regional experience, availability, cost, patient's age, patient's cooperation, side effects of the drug, patient's preferences, especially distance (depending on whether the drug is injected or taken orally) should be considered to choose the drug [11,12]. For example, in terms of cost, deferiprone is less expensive and more available than deferasirox, which has led to its more uses than the other two drugs [13]. Taher et al. in 2010 examined the satisfaction and ease of use of deferasirox with deferoxamine (desferal) among 237 patients and found that patients preferred deferasirox to desferal because of its ease of administration and no need for injection [14].

In patients with thalassemia, taking iron-chelating agents should be started when the ferritin level reaches more than $1000 \mathrm{ng} / \mathrm{ml}$, which usually begins two years after treatment with blood unit transfusion [15]. Deferoxamine at a single dose should be taken by slow intravenous injection when patients develop acute complications due to iron overload. However, deferoxamine is also taken in the case with chronic iron overload at a dose of 4-50 mg/kg per day for 812 hours for 5 to 7 days. Deferoxamine should be adjusted in patients with renal insufficiency, but not in patients with hepatic impairment. However, the administration of deferoxamine may be associated with side effects, such as hives, hot flashes, hypotension, and shock, which are less common in oral forms of iron-chelating agents [16]. Deferiprone, administered orally at a dose of $25 \mathrm{mg} / \mathrm{kg}$ three times a day, does not require dose adjustment in cases with renal or hepatic insufficiency. However, there is a risk of bone marrow suppression and neutropenia and should be discontinued if neutropenia occurs [17]. Deferasirox is another oral iron-chelating agent that is given at a dose of $20 \mathrm{mg} / \mathrm{kg}$ per day and should be adjusted for cases with renal and hepatic insufficiency and also in cases with severe liver and kidney failure, it should not be prescribed. However, it is prescribed once a day, which is an advantage over two other types [18].
Considering the average level of target ferritin in thalassemia patients, studies have shown that the maximum ferritin level for reducing the overall side effects is $2500 \mathrm{ng} / \mathrm{ml}$; however, it is better to be less than $1000 \mathrm{ng} / \mathrm{ml}$ to avoid the effects of increased iron load on the heart and prevent cardiomyopathy [19-21]. In this study, the overall average ferritin level in patients with thalassemia major was $3438 \mathrm{ng} / \mathrm{ml}$ and only half of the patients had a ferritin level of below $2500 \mathrm{ng} / \mathrm{ml}$. By comparing the average ferritin level, which was relatively higher than the target level in various studies, and also that considering that half of the patients had higher ferritin levels than normal, it can be concluded that half of the patients did not receive appropriate control and follow-up and developing complications due to iron overload and failure of various organs are possible.

In this study, no statistically significant difference was observed between iron-chelating agents regarding serum ferritin levels. Several studies have been conducted on these agents but no study was found comparing these three agents. Maggio et al. compared deferoxamine and deferiprone in terms of decreasing ferritin levels, and there was no significant difference between the two drugs in terms of decreasing ferritin levels [22]. Murad et al. compared deferoxamine and deferoxamine in combination with deferiprone, both compounds reduced ferritin levels significantly when taken by patients for 12 months. However, there was no significant difference between the two groups in reducing ferritin levels [23]. Recently, another ironchelating agent called Dexeroyx has been introduced that has not yet routinely entered the pharmaceutical market. In the study by Haji Gholami et al., who compared deferoxamine and this agent, no significant difference was observed in the reduction of serum ferritin levels between these two iron-chelating agents in thalassemia patients [24].

In this study, there was no association between sex and decreased serum ferritin levels following taking the iron-chelating agent. It was supposed that the average serum level may be lower in females due to the menstruation in female patients, but there was no statistically significant difference between the genders in serum ferritin. In a study by Borgna et al. after the discovery of deferoxamine, female thalassemia major patients born in the 1970s and older had lower mortality and morbidity than those born in the 1960s [25]. Hajigholami et al. compared the deferoxamine and dexeroyx iron-chelating agents and found no significant difference in the reduction of ferritin levels between these two agents in both sexes [24]

The study also had limitations; the study conditions, especially nutrition, which plays an important role in changes in patients' serum ferritin levels, were not controlled. Another limitation was the small sample size and differences between the numbers of samples 
in the three groups treated with different ironchelating agents.

Due to the limitations of the descriptive study and the small sample size, it is suggested that a comparison be made between the three drugs of deferoxamine, deferiprone, and deferasirox in future clinical trials study using larger sample size.

\section{Conclusion}

There is no difference between deferoxamine, deferiprone, and deferasirox iron-chelating agents in reducing the ferritin levels of patients with thalassemia and all can be administrated considering the cost, availability, condition, and patient's desire.

Acknowledgments: This study was extracted from research approved by the Research Council of Yasouj University of Medical Sciences. The authors are thankful to the efforts of the staff of the Cooley's ward of Shahid Beheshti Hospital and the Vice Chancellor for Research and Technology of Yasouj University of Medical Sciences, the students who participated in the research and all who contributed to the implementation of this study.

Ethical Permission: This study was registered at the Research Ethics Committee of Yasouj University of Medical Sciences (IR.YUMS.REC.1397.109). The written consent was obtained from the participants.

Conflict of interests: The authors state that there is no conflict of interests.

Authors' contribution: Aria A. (First author), Introduction author/ Original researcher/ Discussion author (16\%); Jokar S. (Second author), Methodologist/ Assistant (10\%); Etemadfar P. (Third author), Introduction author/ Assistant (10\%); Malekzadeh J. (Fourth author), Assistant/ Statistical analyst (10\%); Jannesar R. (Fifth author), Introduction author (10\%); Rohani M. (Sixth author), Assistant/ Discussion author (8\%); Mohammadi T. (Seventh author), Introduction author/ Assistant (7\%); Kharaman F. (Eighth author), Introduction author/Assistant (5\%); Yousefi M. (Ninth author), Methodologist/ Assistant (5\%); Hatamipour S. (Tenth author), Methodologist/ Assistant (5\%); Roozbehi A. (Eleventh author), Introduction author/ Methodologist/ Discussion author (14\%)

Funding: This research was funded by the Vice-Chancellor for Research and Technology of Yasouj University of Medical Sciences.

\section{References}

1- Galanello R, Origa R. Beta-thalassemia. Orphanet J Rare Dis. 2010;5(1):1-15.

2- Rother RP, Bell L, Hillmen P, Gladwin MT. The clinical sequelae of intravascular hemolysis and extracellular plasma hemoglobin: a novel mechanism of human disease. JAMA. 2005;293(13):1653-62.

3- Hill QA, Hill A, Berentsen S. Defining autoimmune hemolytic anemia: a systematic review of the terminology used for diagnosis and treatment. Blood Adv. 2019;3(12):1897-906.

4- Lang N, Reibke R. Durch $\beta$-Thalassaemia minor maskierte autoimmune perniziöse Anämie. DMW-Deutsche Medizinische Wochenschrift. 2007;132(40):2083-4.

5- Hoffbrand AV, Taher A, Cappellini MD. How I treat transfusional iron overload. Blood. 2012;120(18):3657-69. 6- Kasper DL, Fauci AS, Hauser SL, Longo DL, Jameson JL,
Loscalzo J. Harrison's principles of internal medicine. 19th Edition. New York: McGraw Hill; 2015. p. 640-9.

7- Hershko C, Link G, Cabantchik I. Pathophysiology of iron overload. Ann N Y Acad Sci. 1998. 850(1):191-201.

8- Cappellini MD, Cohen A, Piga A, Bejaoui M, Perrotta S, Agaoglu L, et al. A phase 3 study of deferasirox (ICL670), a once-daily oral iron chelator, in patients with $\beta$ thalassemia. Blood, 2006;107(9):3455-62.

9- Allain P, Mauras Y, Chaleil D, Simon P, Ang KS, Cam G, et al. Pharmacokinetics and renal elimination of desferrioxamine and ferrioxamine in healthy subjects and patients with haemochromatosis. Br J Clin Pharmacol. 1987;24(2):207-12.

10- Galanello R, Piga A, Alberti D, Rouan MC, Bigler H, Séchaud R. Safety, tolerability, and pharmacokinetics of ICL670, a new orally active iron-chelating agent in patients with transfusion-dependent iron overload due to $\beta$ thalassemia. J Clin Pharmacol. 2003;43(6):565-72.

11- Aydinok Y, Kattamis A, Viprakasit V. Current approach to iron chelation in children. $\mathrm{Br} \mathrm{J}$ Haematol. 2014;165(6):745-55.

12- Musallam KM, Angastiniotis M, Eleftheriou A, Porter JB. Cross-talk between available guidelines for the management of patients with beta-thalassemia major. Acta Haematol. 2013;130(2):64-73.

13- Maggio A. Light and shadows in the iron chelation treatment of haematological diseases. $\mathrm{Br} \mathrm{J}$ Haematol. 2007;138(4):407-21.

14- Taher A, Al Jefri A, Elalfy MS, Al Zir K, Daar S, Rofail D, et al. Improved treatment satisfaction and convenience with deferasirox in iron-overloaded patients with $\beta$ thalassemia: results from the ESCALATOR trial. Acta Haematol. 2010;123(4):220-5.

15- Sarigianni M, Liakos A, Vlachaki E, Paschos P, Athanasiadou E, Montori VM, et al. Accuracy of magnetic resonance imaging in diagnosis of liver iron overload: a systematic review and meta-analysis. Clin Gastroenterol Hepatol. 2015;13(1):55-63. e5.

16- Borgna-Pignatti C, Marsella $M$. Iron chelation in thalassemia Major. Clin Ther. 2015;37(12):2866-77.

17- Neufeld EJ. Oral chelators deferasirox and deferiprone for transfusional iron overload in thalassemia major: new data, new questions. Blood. 2006;107(9):3436-41.

18- Pennell D J, Porter JB, Cappellini MD, El-Beshlawy A, Chan LL, Aydinok Y, et al. Efficacy of deferasirox in reducing and preventing cardiac iron overload in $\beta$-thalassemia. Blood. 2010;115(12):2364-71.

19- Porter JB, Shah FT. Iron overload in thalassemia and related conditions: therapeutic goals and assessment of response to chelation therapies. Hematol Oncol Clin North Am. 2010:24(6):1109-30.

20- Goel H, Girisha KM, Phadke SR. Long-term efficacy of oral deferiprone in management of iron overload in beta thalassemia major. Hematology. 2008;13(2):77-82.

21- Borgna-Pignatti C, Cappellini MD, De Stefano P, Del Vecchio GC, Forni GL, Gamberini MR, et al. Cardiac morbidity and mortality in deferoxamine-or deferipronetreated patients with thalassemia major. Blood. 2006;107(9):3733-7.

22- Maggio A, D’Amico G, Morabito A, Capra M, Ciaccio C, Cianciulli $P$, et al. Deferiprone versus deferoxamine in patients with thalassemia major: a randomized clinical trial. Blood Cells Mol Dis. 2002;28(2):196-208.

23- Mourad FH, Hoffbrand AV, Sheikh-Taha M, Koussa S, Khoriaty AI, Taher A. Comparison between desferrioxamine and combined therapy with 
193

desferrioxamine and deferiprone in iron overloaded thalassaemia patients. Br J Haematol. 2003;121(1):187-9.

24- Hajigholami A, Ansari H, Honarmand S. Comparing the efficacy of Dexeroyx (Osveral) and Deferoxamine (Desferal) in reducing serum ferritin level in patients with thalassemia major. Middle East J Fam Med.
2018;7(10):218-22.

25- Borgna-Pignatti C, Rugolotto S, De Stefano P, Zhao H, Cappellini MD, Del Vecchio GC, et al. Survival and complications in patients with thalassemia major treated with transfusion and deferoxamine. Haematologica. 2004;89(10):1187-93. 